\title{
CHARAKTERISIERUNG DES MIKROBIELLEN SYMBIOMS VON BLUTEGELN
}

\author{
Stephanie Michel, Nadine Jork, Christian Rockmann, Markus Grohme, Philipp Franke, Detlef Menzel, Marcus Frohme
}

\section{Zusammenfassung}

Die medizinischen Blutegel, Hirudo medicinalis und Hirudo verbana, werden wegen ihrer kurativen Wirkung in der Humanmedizin angewandt. Während des Blutsaugens injizieren sie über ihren Speichel eine Vielzahl bioaktiver, derzeit noch unbekannter Moleküle. Eine vollständige Aufklärung aller Inhaltsstoffe mit Wirkmechanismen ist für die Entwicklung von neuen Pharmaka von großem Interesse. Vor diesem Hintergrund wurden verschiedene Organe beider Arten auf ihre Besiedlung durch symbiontische Bakterien untersucht. Dazu wurden die Bakterien zunächst unter geeigneten Bedingungen kultiviert und mittels biochemischer Methoden charakterisiert. Die Identifizierung der Symbionten erfolgte durch Polymerasekettenreaktion (PCR) und Sequenzierung der 16S rDNA. Die biochemischen Tests ergaben, dass die kultivierbaren Bakterien Amylase positiv, Gram negativ und Ornithin Decarboxylase negativ sind. Mit Hilfe von datenbankgestützten Analysen der 16S rDNA-Sequenzen konnte Aeromonas veronii biovar sobria nachgewiesen werden. Hochdurchsatzsequenzierungen der gesamtgenomischen DNA des Bakteriums aus $H$. medicinalis zeigten deutliche Abweichungen zum Referenzgenom von $\mathrm{Ae}$ romonas veronii $\mathrm{B} 565$.

\section{Abstract}

The medical leeches, Hirudo medicinalis and Hirudo verbana, are increasingly used in human medicine because of their curative effect. During bloodsucking, they inject a variety of bioactive molecules via their saliva of which most are still unknown. The investigation of all contents and their mechanisms of action are of great interest, especially for the development of new pharmaceutical products. Against this background, the colonization of various organs of both species by symbiotic bacteria was examined. For this purpose, the bacteria were cultivated under suitable conditions and characterized using biochemical methods. The identification of the symbionts was carried out by polymerase chain reaction (PCR), supported by sequencing of the 16S rDNA. The biochemical tests indicated that the culturable bacteria are amylase positive, gram negative and ornithine decarboxylase negative. The occurrence of Aeromonas veronii biovar sobria could be proved by database analyses of the 16S rDNA. High-throughput sequencing of the genomic DNA showed significant differences from the already known reference genome of Aeromonas veronii B565.

\section{»I. EINFÜHRUNG}

Der medizinische Blutegel, Hirudo medicinalis, wurde bereits in der Antike zum therapeutischen Aderlass eingesetzt. Aufgrund der antikoagulativen, antiinflammatorischen, immunisierenden und analgetischen Wirkung hat sich die Blutegeltherapie vor allem in der Mikrochirurgie, Naturheilkunde sowie für die Behandlung von Thrombosen und Embolien etabliert (Wells et al. 1993: 183). Da $H$. medicinalis in Europa fast ausgestorben ist, werden heutzutage entweder Zuchttiere oder die in Kleinasien lebenden Egel namens $H$. verbana zu therapeutischen Zwecken verwendet. Während des Blutsaugens, aber auch beim Loslassen, injiziert der Egel über seinen Speichel eine Vielzahl von medizinisch nützlichen, bioaktiven Molekülen. Bisher sind nur acht Inhaltsstoffe bekannt. Dazu zählen unter anderem das Hirudin und das Calin, welche die Koagulation des Blutes hemmen (Zaidi et al. 2011: 437). Für die Anwendung in der Humanmedizin rücken die Blutegel zunehmend in den arzneimittelrechtlichen Fokus. Seit dem Jahr 2004 sind die Tiere zwar gemäß § 2 Absatz 1 des Arzneimittelgesetzes als Arzneimittel zugelassen, jedoch ist der Egel an sich vergleichsweise schlecht definiert. So verfügen die Tiere über symbiontische Bakterien, welche sich obligat im Verdauungstrakt (Intestinum) befinden, wobei die mikrobielle Flora des Blutegels im Vergleich zu anderen Tieren relativ einfach aufgebaut zu sein scheint (Graf et al. 2006: 365). Bisher konnten nur zwei Bakterien, Aeromonas veronii biovar sobria und Bakterien der Gattung Rikenella, im Verdauungstrakt des Egels identifiziert werden (Graf 1999: 1). Neue Erkenntnisse über diese symbiontischen Bakterien könnten die Arzneimittelsicherheit in Hinblick auf eine standardisierte Laboraufzucht verbessern und dazu beitragen, die physiologischen Zusammenhänge im Egel besser zu verstehen. Letzteres ist vor allem für die rekombinante Herstellung von neuen therapeutischen Proteinen von Bedeutung. Die Symbiose zwischen dem Wirt
H. medicinalis und dem Symbionten $A$. veronii biovar sobria ist noch weitgehend unverstanden. Es besteht die Annahme, dass der Symbiont den Wirt mit Nährstoffen versorgt (Graf 2010) und dass Aeromonas an der Verdauung des aufgenommenen Blutes beteiligt ist, zumal bislang keine an der Verdauung beteiligten Egel-Enzyme nachgewiesen werden konnten. Der Symbiont hingegen ist für seine beta-hämolytischen Fähigkeiten bekannt und könnte die Lyse der Erythrozyten übernehmen (Kozaki et al. 1989: 1782). Untersuchungen ergaben, dass der Wirt wiederum Proteasen ins Intestinum absondert, welche die Beta-Hämolyse inhibieren und somit die Speicherung der Erythrozyten ermöglichen (Roters et Zebe 1992: 85). Darüber hinaus zeigten weiterführende Studien, dass Aeromonas die Proliferation von nicht symbiontischen Bakterien im Kropf des Wirtes inhibiert (Indergand et Graf 2000: 4735).

Ziel dieser Arbeit war die Isolierung und Charakterisierung symbiontischer 
Bakterien aus dem Verdauungstrakt des medizinischen Blutegels $H$. medicinalis. Parallel wurden die symbiontischen Bakterien aus $H$. verbana, welcher ebenfalls zu medizinischen Zwecken eingesetzt wird, extrahiert und bestimmt. Zur Isolierung der Bakterien wurden die Egel zunächst präpariert, der Inhalt des Verdauungstraktes (Intestinums) sowie die Speicheldrüsen (Glandulae salivariae) entnommen und auf Stärke-AmpicillinAgar ausplattiert. Anschließend wurden die Kolonien mithilfe geeigneter biochemischer Methoden, wie Amylase-, Gram- und Decarboxylase-Tests untersucht. Charakteristische Kolonien wurden in Flüssigmedien überführt und die Gesamt-DNA isoliert. Zur eindeutigen Identifizierung der Isolate wurden Amplifikate des 16S rDNA Gens mittels konventioneller Polymerasekettenreaktion (PCR) erstellt (Keller et al. 2010: 755) und sequenziert. Ziel war außerdem eine Hochdurchsatzsequenzierung der gesamtgenomischen DNA.

\section{» II. MATERIAL UND METHODEN}

Die Isolierung der symbiotischen Bakterien erfolgte aus Hirudo medicinalis und Hirudo verbana, welche von der BioRepro GmbH Potsdam bereitgestellt wurden. Dazu wurden Teile der Speicheldrüsen sowie des Verdauungstraktes herauspräpariert, auf Stärke-AmpicillinAgar (10 g/l Trypton, 1 g/l Hefe-Extrakt, $5 \mathrm{~g} / \mathrm{l}$ Natriumchlorid, 0,025 g/l Phenolrot, 15 g/l Agar, $10 \mathrm{~g} / \mathrm{l}$ Stärke, $\mathrm{pH} 7,4$ ) ausplattiert und über Nacht bei $29^{\circ} \mathrm{C}$ inkubiert. Charakteristische EinzelkoIonien wurden isoliert, ggf. auf Platte oder in LB-Medium (8 g/l Trypton, $4 \mathrm{~g} / \mathrm{l}$ Hefe-Extrakt, $8 \mathrm{~g} / \mathrm{l}$ Natriumchlorid, $\mathrm{pH}$ $7,0)$ weiter kultiviert, um mittels biochemischer und molekularbiologischer Methoden untersucht werden zu können.

Zur Bestimmung der Amylaseaktivität wurden Stärke-Ampicillin-Agarplatten mit Bakterienkolonien nach einer Inkubationszeit von drei Wochen bei $4{ }^{\circ} \mathrm{C}$ mit Lugolscher Lösung überdeckt, eine Minute bei Raumtemperatur inkubiert und anschließend mit Wasser gewaschen.

Die Untersuchung auf Gramverhalten erfolgte, indem $10 \mu$ l einer LB-Übernachtkultur auf einen Objektträger überführt und mit $30 \mu \mathrm{l} 3 \%$ iger $\mathrm{KOH}$-Lösung suspendiert wurden. Nach einer
Inkubationszeit von fünf Minuten bei Raumtemperatur wurde die Viskosität der Suspension mit einer Impföse kontrolliert.

Zur Bestimmung der Aeromonas Subspezien wurde ein Ornithin-Decarboxylase-Test durchgeführt. Dazu wurden Einzelkolonien von Stärke-AmpicillinAgarplatten gepickt und in jeweils $4 \mathrm{ml}$ Ornithin-Decarboxylase-Medium (5 g/l Trypton, $3 \mathrm{~g} / \mathrm{l}$ Hefe-Extrakt, $1 \mathrm{~g} / \mathrm{l}$ Glukose, 0,02 g/l Bromkresolpurpur, $5 \mathrm{~g} / \mathrm{l}$ Ornithin, pH 6,3) überführt. Die Reaktionsansätze wurden anschließend mit PlusOne DryStrip Cover Fluid (GE Healthcare) bedeckt und für $24 \mathrm{~h}$ bei $29^{\circ} \mathrm{C}$ unter Schütteln inkubiert. Die Decarboxylase-Fähigkeit der Bakterien wurde optisch anhand des mitgeführten $\mathrm{pH}$-Indikators Bromkresolpurpur bestimmt.

Zur Extraktion der Gesamt-DNA wurden $2 \mathrm{ml}$ Übernachtkultur für fünf Minuten bei 13000 x g zentrifugiert. Der Überstand wurde verworfen und das Pellet in $500 \mu \mathrm{l}$ TE-Puffer $(50 \mathrm{mM}$ TRIS-HCL, 1 mM EDTA, pH 8,0) resuspendiert. Nach erneuter Zentrifugation für fünf Minuten bei $13000 \times \mathrm{g}$ wurde das Pellet in $500 \mu$ l eiskaltem TES-Puffer $(50 \mathrm{mM}$ TRIS-HCL, 100 mM EDTA, 25\% Saccharose) resuspendiert und für eine Stunde auf Eis inkubiert. Nach der Zugabe von $50 \mu$ l Lysozym (AppliChem) erfolgte eine Inkubation für 30 Minuten bei $37^{\circ} \mathrm{C}$. Anschließend wurden $50 \mu \mathrm{l} 20 \%$ iges SDS (Endkonzentration 2\%) und $15 \mu \mathrm{l}$ Proteinase K (AppliChem) zugegeben. Nach einer Inkubation über Nacht bei $37^{\circ} \mathrm{C}$ wurde ein Volumen Phenol-Chloroform-Isoamylalkohol ( $\mathrm{PCl}$ ) hinzugegeben, intensiv geschüttelt und für zehn Minuten bei $13000 \times \mathrm{g}$ und $4^{\circ} \mathrm{C}$ zentrifugiert. Die obere wässrige Phase wurde in ein neues Reaktionsgefäß überführt und die $\mathrm{PCl}$-Aufreinigung wiederholt. Anschließend wurde ein Volumen Chloroform-Isoamylalkohol zugegeben, intensiv geschüttelt und für zehn Minuten bei $13000 \times \mathrm{g}$ und $4{ }^{\circ} \mathrm{C}$ zentrifugiert. Die obere wässrige Phase wurde in ein neues Reaktionsgefäß überführt und die DNA durch Zugabe des 0,7-fachen Volumens Isopropanol gefällt. Nach einer Zentrifugation von 30 Minuten bei $13000 \times \mathrm{g}$ und $4{ }^{\circ} \mathrm{C}$ wurde das Pellet zweimal mit 70\%igem Ethanol gewaschen. Das getrocknete DNA-Pellet wurde mit $30 \mu \mathrm{LEPC}-\mathrm{H} 2 \mathrm{O}$ resuspendiert. Die Konzentrationsmessung der
DNA erfolgte mithilfe des NanoDrop ND-1000 Spektrophotometer (Peqlab) durch Absorptionsmessung bei einer Wellenlänge von $\lambda=260 \mathrm{~nm}$. Des Weiteren wurde der Absorptionsquotient A260/A280 herangezogen, um den Reinheitsgrad der DNA zu bestimmen. Die extrahierte Gesamt-DNA wurde anschließend mittels Gelelektrophorese bei $75 \mathrm{~V}$ für 35 Minuten in einem $1 \%$ Agarosegel und 1x TAE Puffer aufgetrennt. Das Gel wurde in einer Ethidiumbromidlösung $(0.5 \mu \mathrm{g} / \mathrm{ml})$ gefärbt und unter UV-Licht analysiert.

Die Amplifikation des variablen Bereichs der 16S rDNA wurde mithilfe einer PCR durchgeführt. Die Primer wurden der Publikation von Graf entnommen (Graf 1999: 1). Der Mastermix bestand aus $0.2 \mathrm{mM}$ dNTPs, $0.25 \mu \mathrm{M}$ des Forward Primers und des Backward Primers, 0.2 U Taq Polymerase (DreamTaq ${ }^{\mathrm{TM}}$, Fermentas) und $200 \mathrm{ng}$ Ziel-DNA in einem Volumen von $50 \mu \mathrm{l}$ 1x Reaktionspuffer (DreamTaq $^{\mathrm{TM}}$, Fermentas). Die Amplifikationsbedingungen waren wie folgt: $95^{\circ} \mathrm{C}$ für zwei Minuten, gefolgt von 30 Zyklen bei $95^{\circ} \mathrm{C}$ für $30 \mathrm{~s}, 57^{\circ} \mathrm{C}$ für 15 $\mathrm{s}$ und $72{ }^{\circ} \mathrm{C}$ für $30 \mathrm{~s}$. Amplifikate in der Größe von 599bp wurden elektrophoretisch aufgetrennt und detektiert (wie oben beschrieben). Die PCR-Produkte wurden anschließend mittels QIAquick PCR purification kit (Quiagen) aufgereinigt. Dabei wurde nach Angaben des Herstellers vorgegangen und in $30 \mu \mathrm{l}$ Elutionspuffer eluiert. Die Konzentration und Reinheit der Amplifikate wurden mit dem Nanodrop ND-1000 Spektrophotometer (Peqlab) bestimmt. Die Sequenzierung der amplifizierten 16S rDNA wurde durch den Anbieter StarSEQ (Mainz) durchgeführt. Diese erfolgte vom 5'-Ende mit Hilfe des ForwardPrimers (Graf 1999: 1).

Weiterhin wurden whole-genome-shotgun Sequenzierungen der Bakterien aus H. medicinalis mit GS Junior (Roche) und Ion Torrent (Life Technologies) durchgeführt. Für die referenzbasierten Assemblies wurden GS Reference Mapper 2.7 von 454 Life Sciences (http://454. com/products/analysis-software/index. asp) sowie MIRA 3.9.4 (Chevreux et al. 2009: 1147) verwendet. Die Reads der 454 und Ion Torrent Sequenzierungen wurden hierbei auf das Referenzgenom Aeromonas veronii B565 (Li et al. 2011: 3389) gemappt. Eine de novo 
Assemblierung erfolgte mit GS De Novo Assembler 2.7 von 454 Life Sciences. Mit der minimus2-Pipeline (http://sourceforge.net/apps/mediawiki/amos/ index.php?title=Minimus2) des AMOS Paketes wurden die Datensets aus dem Mappingassembly und dem de novo Assembly zusammengeführt. Mithilfe des Referenzgenoms wurden die Contigs mit Mauve (Rissman et al. 2009: 2071) sortiert.

\section{»III. ERGEBNISSE}

Zur Charakterisierung des mikrobiellen Symbioms in medizinischen Blutegeln
Nach einem Kultivierungszeitraum von drei Wochen bei $4{ }^{\circ} \mathrm{C}$ wurden die Bakterien auf ihre Fähigkeiten untersucht, Stärke zu hydrolysieren. Dazu wurden die Stärke-Ampicillin-Agarplatten mit Lugolscher Lösung angefärbt. Anhand der farblosen Zonen, welche sich um die Kolonien bildeten, konnten in den Speicheldrüsen- und Verdauungstraktproben beider Egel Amylase-positive Bakterien nachgewiesen werden.

Weiterhin zeigten alle Bakteriensuspensionen nach der Behandlung mit $\mathrm{KOH}$ eine Zunahme in ihrer Viskosität, was auf die Lysierung der Zellwände schließen lässt. Somit konnten alle Isolate als Gram-negativ identifiziert werden.

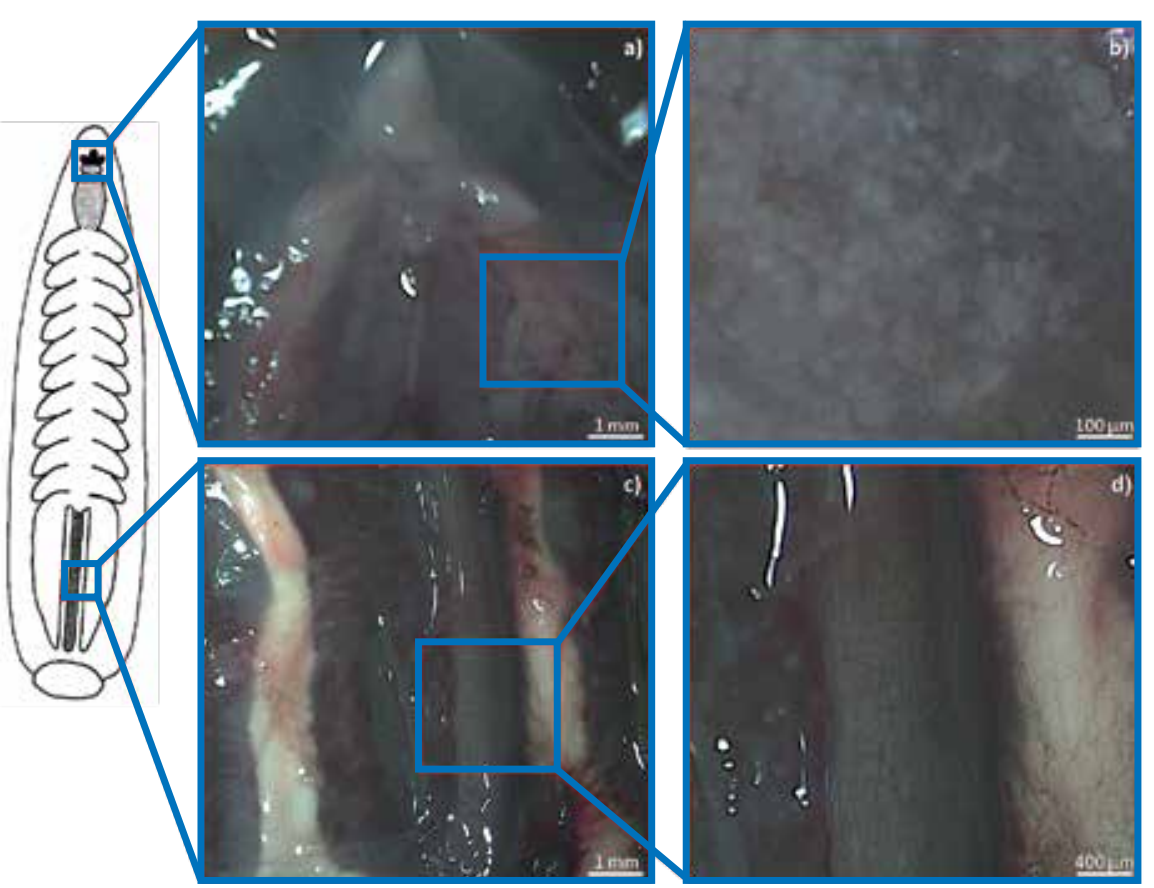

Abb. 1) Ventrale Präparation der Speicheldrüsen (Glandulae salivariae) und des Verdauungstraktes (Intestinum) von H. medicinalis. (H. verbana analog) im Schema. Foto links oben: Übersicht der Kieferplatten mit angrenzenden Speicheldrüsen. Foto rechts oben: Speicheldrüsengewebe. Foto links unten: Übersicht Verdauungstrakt mit angrenzender Leibeshöhle (Peritoneum). Foto rechts unten: Gefüllter Verdauungstrakt.
Zur Bestimmung der Aeromonas Subspezien wurde ein Ornithin-Decarboxylase-Test im Flüssigmedium durchgeführt. Sind die Bakterien in der Lage, die im Medium enthaltene Glukose zu fermentieren, entsteht ein saures $\mathrm{Ne}$ benprodukt, woraus ein Farbumschlag von violett zu gelb resultiert. Besteht die Fähigkeit, Ornithin zu decarboxylieren, werden alkalische Nebenprodukte (Putrescine) gebildet und das Medium bleibt violett gefärbt. Während $A$. veronii biovar veronii die Fähigkeit besitzt Ornithin zu decarboxylieren, ist $A$. veronii biovar sobria negativ für diese Reaktion. Nach einer Inkubation von $24 \mathrm{~h}$ bei $29^{\circ} \mathrm{C}$ konnte sowohl bei den isolierten Bakterien aus $H$. medicinalis als auch bei denen aus $H$. verbana ein Farbumschlag von violett nach gelb beobachtet werden. Während sich bei den Kulturen aus $H$. verbana das gesamte Medium verfärbte, ist bei denen aus $H$. medicinalis nur ein Teil des Mediums in gelb umgeschlagen (Abb. 2). Demnach fermentieren die Bakterien beider Egel die im Flüssigmedium enthaltende Glukose. Sie besitzen nicht die Fähigkeit, Ornithin zu decarboxylieren und können demzufolge als $A$. veronii biovar sobria identifiziert werden.

Die Gesamt-DNA der Bakterien aus $H$. medicinalis und $H$. verbana wurde mittels Phenol Chloroform Isoamylalkohol und anschließender Isopropanolfällung isoliert und elektrophoretisch aufgetrennt. Dabei konnte eine DNA Bande oberhalb der 20000bp nachgewiesen werden, welche hochmolekularer genomischer DNA entspricht. Zudem waren zwei DNA Banden oberhalb von 1500bp und 1000bp zu erkennen,

wurden aus den Speicheldrüsen und dem Verdauungstrakt von $H$. medicinalis und $H$. verbana Proben entnommen (Abb. 1), auf Stärke-Ampicillin-Agar ausgestrichen und über Nacht bei $29^{\circ} \mathrm{C}$ inkubiert. Bereits nach $12 \mathrm{~h}$ konnte sowohl bei den Bakterienausstrichen von $H$. medicinalis als auch bei denen von $H$. verbana ein Wachstum von großen, runden, gelb bis grauweißen Kolonien beobachtet werden. Die vorliegende Ampicillin-Resistenz sowie die phänotypischen Merkmale deuteten auf die Bakteriengattung Aeromonas hin (Imziln 2001: 796; Glünder und Reetz 2005: 223).

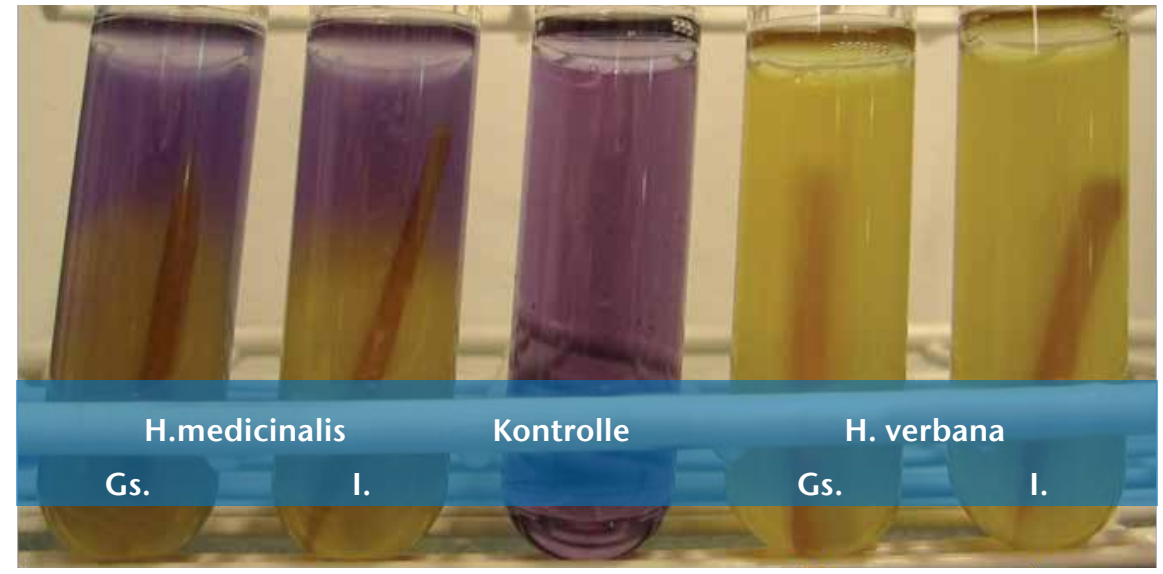

Abb. 2) Ornithin-Decarboxylase Test (Farbumschlag von violett nach gelb). Farbumschlag des Mediums bei Ornithin-Decarboxylase-negativen Bakterien, isoliert aus den Speicheldrüsen (Glandulae salivariae = Gs.) bzw. dem Verdauungstrakt (Intestinum = I.). von H. medicinalis und $\mathrm{H}$. verbana. 


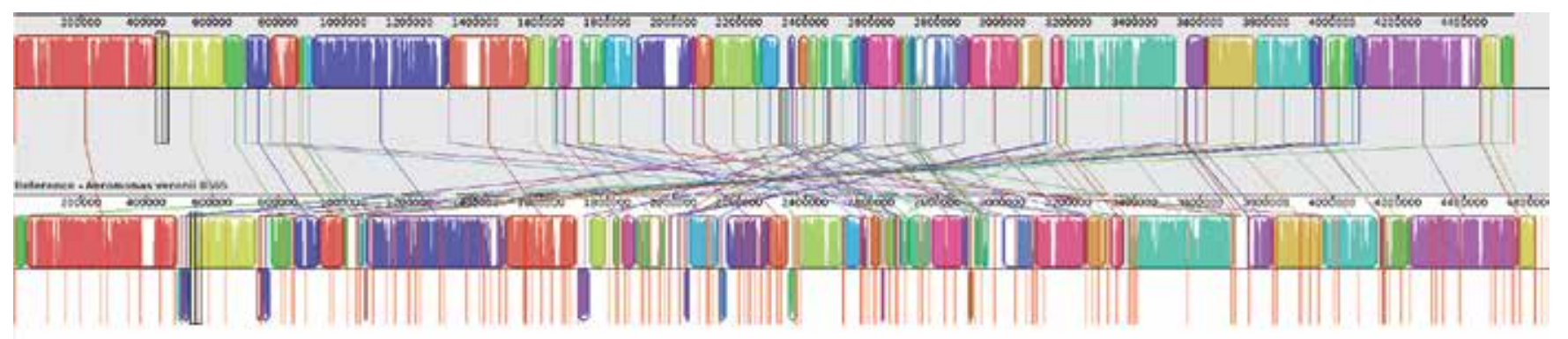

Abb. 3) Sequenzvergleich des publizierten Aeromona veronii Stamm B565 (oben) mit dem Assembly des sequenzierten Bakteriums aus H. medicinalis.

welche laut Nawaz et al. charakteristisch für niedermolekulare Plasmid DNA in $A$. veronii sind (Nawaz et al. 2006: 6461). Mithilfe der Sequenzierung der 16S rDNA durch den externen Anbieter StarSEQ (Mainz) konnten alle Amplifikate der Gattung $A$. veronii zugeordnet werden. Anhand der 16S rDNA-Sequenz konnte jedoch nicht zwischen den beiden Subspezien $A$. veronii biovar veronii und $A$. veronii biovar sobria unterschieden werden.

Die referenzbasierten Assemblies mit GS Reference Mapper bzw. MIRA zeigten eine Abdeckung der Referenzsequenz von $81 \%$ bzw. $82 \%$ (4265 Contigs) aus einem de novo Assembly resultierten 282 Contigs. Das Zusammenführen beider Datensets mit minimus 2 reduzierte die Anzahl der Contigs auf 159, welche mit Mauve sortiert wurden (Abb. 3).

\section{»IV.DISKUSSION}

Mithilfe biochemischer Methoden konnten Amylase-positive, Gram-negative und Ornithin-Decarboxylase-negative Bakterien in den Speicheldrüsen und dem Verdauungstrakt von $H$. medicinalis und $H$. verbana nachgewiesen werden. Zusammen mit den Ergebnissen der 16S rDNA-Sequenzierung konnte Aeromonas veronii biovar sobria als Symbiont identifiziert werden. Auffällig war jedoch, dass die isolierten Bakterien aus $\mathrm{H}$. verbana und $H$. medicinalis unterschiedliche Glukose-Fermentationsaktivitäten zeigten. Somit ist nicht auszuschließen, dass es sich bei den aus beiden Egelarten isolierten Bakterien um unterschiedliche Subspezien des Bakteriums A. veronii handeln könnte, obwohl die Ergebnisse der $16 \mathrm{~S}$ rDNA-Sequenzierung beider Symbionten auf das $A$. veronii biovar sobria hindeuten. Es wäre denkbar, dass sich die Aeromonaden infolge der standardisierten Laboraufzucht differenziert haben. Wie bereits von Graf et al. beschrieben, scheint die mikrobielle Flora des Blutegels im Vergleich zu anderen Tieren relativ einfach aufgebaut zu sein (Graf et al. 2006: 365). Bisher konnten nur zwei Bakterien, Aeromonas veronii biovar sobria und Bakterien der Gattung Rikenella, im Verdauungstrakt des Egels identifiziert werden (Graf 1999: 1). Die Ergebnisse der Sequenzierung der Bakterien aus $H$. medicinalis zeigten, dass diese in ihrer Genomsequenz nur zu ca. 80\% mit dem publizierten Aeromona veronii Stamm B565 übereinstimmen. Ein gesamtgenomischer Sequenzieransatz auf größere Fragmente wird die vorhandenen Lücken in der Sequenz schließen können. Im Vergleich mit dem noch zu analysierenden Genom aus $H$. verbana wird deutlich werden, worin die Unterschiede beider Symbionten hinsichtlich der DNA und biochemischer Ebene bestehen. 
LITERATUR

Chevreux B., Pfisterer T., Drescher B., Driesel A. J., Müller W. E. G., Wetter T., Suhai S. (2004): Using the miraEST Assembler for Reliable and Automated mRNA Transcript Assembly and SNP Detection in Sequenced ESTs. In: Genome Research. 2004; 14: 1147-1159.

Delcher A. L., Phillippy A., Carlton J., Salzberg S.L. (2002): Fast Algorithms for Large-scale Genome Alignment and Comparision. In: Nucleic Acids Research. 2002; 30: 2478-2483.

Glünder G. und Reetz G. (2005): Aeromonas-Septikämie. Hrsg. v. Otfried Siegmann u. Ulrich Neumann: Kompendium der Geflügelkrankheiten. Schlütersche Verlag. 2005; 6 . Auflage. Kapitel 3, 223.

Graf J. (1999): Symbiosis of Aeromonas veronii biovar sobria and Hirudo medicinalis, the medicinal leech: a novel model for digestive tract associations. In: Infect. Immun. 1999; 67: 1-7.

Graf J., Kikuchi Y., Rio RV. (2006): Leeches and their microbiota: naturally simple symbiosis models. In: Trends Microbiol. 2006; 14: 365-371.

Graf I. (2010): The Digestive-Tract Symbiosis of Hirudo verbana, the Medicinal Leech. http://web.uconn.edu/ mcbstaff/graf/AvHm/AvHmmain.htm.

Imziln B. (2001): Occurrence and antibiotic resistance of mesophilic Aeromonas in three riverine freshwaters of Marrakech, Morocco. In: Scientific World Journal. 2001; 1:796-807.

Indergand S., Graf J. (2000): Ingested Blood Contribute to the Specificity of the Symbiosis of Aeromonas veronii biovar Sobria and Hirudo medicinalis, the Medicinal Leech. Appl. Environ. Microbiol. 2000; 66(11): 4735-41.

Keller P. M., Hombach M., Bloemberg G. V. (2010): 16SrRNA-Gen-basierte Identifikation bakterieller Infektionen. In: BIOspectrum. 2010; 16: 755-758.

Kozaki S., Asao T., Kamata Y., Sakaguchi G. (1989): Characterization of Aeromonas sobria hemolysin by use of monoclonal antibodies against Aeromonas hydrophila hemolysins. In: J. Clin. Microbiol. 1989; 27(8): 1782-1786.

Li Y., Liu Y., Zhou Z., Huang H., Ren Y., Zhang Y., Li G., Zhou Z., Wang L. (2011): Complete Genome Sequence of Aeromonas veronii Strain B565. In: Journal of Bacteriology. 2011; 193: 3389-3390.

Nawaz M., Sung K., Saeed A., Khan S. A., Khan A. A., Steele R. (2006): Biochemical and Molecular Characterization of Tetracycline-Resistant Aeromonas veronii Isolates from Catfish. In: Appl. Environ. Microbiol. 2006; 72: 6461-6466.

Rissman A. I., Mau B., Biehl B. S., Darling A. E.,

Glasner J. D., Perna N. T. (2009): Reordering contigs of draft genomes using the Mauve aligner. In: Bioinformatics. 2009; 25: 2071-2073.

Roters F. J., Zebe E. (1992): Protease inhibitors in the alimentary tract of the medicinal leech Hirudo medicinalis: in vivo and in vitro studies. In: J. Comp. Physiol. 1992; B 85-92.

Wells M. D., Manktelow R. T., Boyd J. B., Bowen V. (1993): The medical leech: an old treatment revisited. In: Microsurgery. 1993; 14: 183-186.

Zaidi S. M., Jamil S. S., Sultana A., Zaman F., Fuzail M. (2009): Safety and efficacy of leeching therapy for symptomatic knee osteoarthritis using Indian medicinal leech, In: Indian Journal of Traditional Knowledge. 2009; 8(3): 437-442.

\section{AUTOREN}

Stephanie Michel*

M. Sc. der Biosystemtechnik/Bioinformatik

Molekulare Biotechnologie und Funktionelle Genomik

TH Wildau [FH]

smichel@th-wildau.de

Nadine Jork*

M. Sc. der Biosystemtechnik/Bioinformatik

Molekulare Biotechnologie und Funktionelle Genomik

TH Wildau [FH]

njork@th-wildau.de

\section{Christian Rockmann}

M. Sc. der Biosystemtechnik/Bioinformatik

Molekulare Biotechnologie und Funktionelle Genomik TH Wildau [FH]

crockman@th-wildau.de

\section{Markus Grohme}

Diplom-Biologe

Molekulare Biotechnologie und Funktionelle Genomik TH Wildau [FH]

mgrohme@th-wildau.de

\section{Philipp Franke}

M. Sc. der Biosystemtechnik/Bioinformatik

Molekulare Biotechnologie und Funktionelle Genomik TH Wildau [FH]

pfranke@th-wildau.de

\section{Detlef Menzel}

BioRepro $\mathrm{GmbH}$, Potsdam

detlef.menzel@biorepro.de

Prof. Dr. Marcus Frohm

Molekulare Biotechnologie und Funktionelle Genomik TH Wildau [FH]

mfrohme@th-wildau.de 